\title{
Identification of Schistosoma mansoni Infection in a Nonhuman Primate from St. Kitts More than 50 Years after Interruption of Human Transmission
}

\author{
Jennifer K. Ketzis, ${ }^{1 \star} †$ Manigandan Lejeune ${ }^{2} \dagger$ lan Branford, ${ }^{1}$ Amy Beierschmitt, ${ }^{3}$ and Arve Lee Willingham ${ }^{1}$ \\ ${ }^{1}$ One Health Center for Zoonoses and Tropical Veterinary Medicine, Ross University School of Veterinary Medicine, Basseterre, St. Kitts and Nevis; \\ ${ }^{2}$ Department of Population Medicine and Diagnostic Sciences, Animal Health Diagnostic Center, College of Veterinary Medicine, Cornell \\ University, Ithaca, New York; ${ }^{3}$ Behavioural Science Foundation, Estridge Estate, St. Kitts and Nevis
}

\begin{abstract}
Transmission of Schistosoma mansoni was interrupted on St. Kitts, a Caribbean island, in the 1950s. With no reported cases since that time and most Biomphalaria spp. snail populations eliminated based on surveys in the 1970s, S. mansoni has been considered eliminated on St. Kitts. In 2019, S. mansoni eggs were found in an African green monkey (Chlorocebus aethiops sabaeus) that originated from St. Kitts. Nonhuman primate (NHP) infections have been considered incidental to human infections, with infections in NHPs resolving with the elimination of $S$. mansoni in the human population. An NHP with S. mansoni infection suggests that the NHP may be able to maintain a reservoir sylvatic cycle. Alternatively, S. mansoni transmission was not eliminated or S. mansoni has been reintroduced to St. Kitts. The occurrence of an infected NHP from St. Kitts supports the need for continuous monitoring in areas where S. mansoni is considered eliminated.
\end{abstract}

Schistosomiasis, a neglected tropical disease, is caused by blood trematodes of the genus Schistosoma. These trematodes have an indirect life cycle with aquatic snails serving as intermediate hosts and people as the final host. Schistosoma mansoni, the predominate species that causes intestinal schistosomiasis and uses snails of the genus Biomphalaria as the intermediate host, primarily occurs in Africa with introductions via the slave trade to areas of South America and the Caribbean. ${ }^{1}$ Efforts to interrupt transmission and eliminate the parasite are going on in endemic areas with mass drug administration of praziquantel, improvements in water systems, and molluscicide treatments or use of competitive snail species to decrease the Biomphalaria spp. populations. ${ }^{1-4}$

On St. Kitts, in the West Indies, S. mansoni was a significant infectious disease with approximately $25 \%$ of the population infected in the 1930s. ${ }^{5,6}$ Prevalence was highest in the villages of West Farm, Boyds, Old Road, and Cayon, all located near permanent and semipermanent rivers with sources in the mountainous rain forest in the center of the island (Figure 1). ${ }^{5-7}$ In the 1950s, there was natural abatement of S. mansoni infections through changes in water supply, diversion or containment of the rivers through a piping system, and snail elimination campaigns. ${ }^{5}$ In the 1970 s, there were no human cases, and examined snail populations, which were limited to Fountain River, were not infected. ${ }^{5,7,8}$ Therefore, S. mansoni transmission was considered interrupted on St. Kitts in the 1950s and eliminated in the 1970s.

On Caribbean islands and in localities such as Antigua, Dominican Republic, Guadeloupe, Martinique, Montserrat, Puerto Rico and Saint Lucia, where transmission is considered interrupted, there are efforts to confirm that S. mansoni has been eliminated. ${ }^{1-3,6,9,10}$ Given St. Kitts's status of already eliminated, it has not been included in these programs, and there are no ongoing surveillance programs. However, human stool specimens are examined using direct smear or formalin

*Address correspondence to Jennifer K. Ketzis, One Health Center for Zoonoses and Tropical Veterinary Medicine, Ross University School of Veterinary Medicine, P.O. Box 334, Basseterre, St. Kitts and Nevis. E-mail: jketzis@rossu.edu

†These authors contributed equally to this work. sedimentation in suspect helminth infections (e.g., Trichuris trichiura) and for food handler annual health certificates. No $S$. mansoni infections have been reported in these examinations (personal communication with diagnostic technicians at the Joseph N. France General Hospital and Avalon Labs based on records from approximately the last 8 years, the primary public and private laboratories on St. Kitts).

Although people are the definitive final host for S. mansoni in the Caribbean, rodents and nonhuman primates (NHPs) can be infected. ${ }^{8,11-13}$ There is some uncertainty regarding the role of rodents, specifically rats, in perpetuating a sylvatic cycle; however, infection in NHPs has been considered secondary to human infections, with elimination in the human population resulting in elimination in the NHP population. ${ }^{5,8}$ On St. Kitts, African green monkeys (C. a. sabaeus) were introduced over 350 years ago, and populations also exist on Barbados, St. Maarten (St. Martin), and Tortola. ${ }^{14}$ For many years, interactions with people and the NHP population on St. Kitts were limited, with the NHPs predominately located in the mountainous region of the island and adventuring to coastal areas to predate on food crops. ${ }^{14,15}$ In 1928, when S. mansoni was prevalent on St. Kitts, five of seven NHPs examined were positive. ${ }^{10}$ In the 1960 s, approximately 50 NHPs from St. Kitts were examined for $S$. mansoni, with all being negative. ${ }^{16}$ The lack of positive NHPs was attributed to the natural abatement of S. mansoni on St. Kitts and the need for human transmission to maintain infection in NHPs.

Over the last 30 years, the NHP population has increased in size and their interactions with people have increased. ${ }^{14,15}$ Their range is no longer limited to the mountains with NHP troops now resident in many villages on St. Kitts. Nonhuman primates are considered a nuisance species by many, and up to $60 \%$ of food crop destruction is attributed to them. Troops that have taken up residence in farming areas can be trapped and relocated, euthanized, or transferred to one of the research facilities on the island. Those transferred to one of the research facilities are tested frequently for endoparasites. Tests ranging from double centrifugation with Sheather's sugar flotation solution, zinc sulfate with/without staining for protozoa, direct smear, to sedimentation are conducted on St. Kitts and during quarantine when the NHPs are transported to 


\section{ATLANTIC OCEAN}

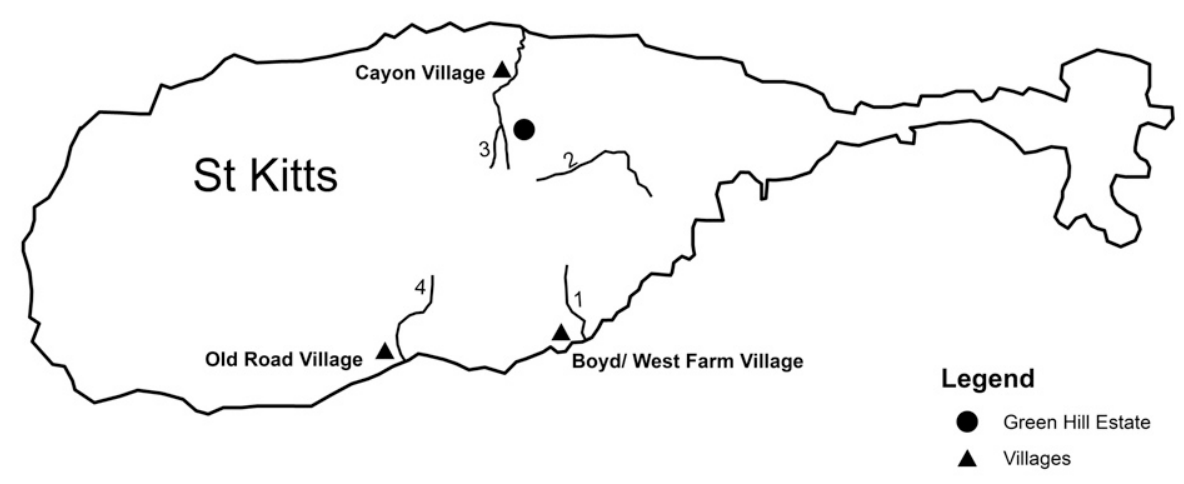

CARIBBEAN SEA

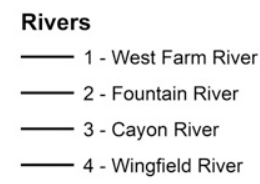

1 centimeter $=40$ meters

$\begin{array}{lll}0 & 0.05 \quad 0.1\end{array}$ 0.2 Miles

FIGURE 1. Map of St. Kitts indicating villages where schistosomiasis was prevalent in the 1930s and the relevant permanent or semipermanent rivers in the 1930s leading to these villages; the Fountain River, the location of Biomphalaria spp. snails in the 1970s; and Green Hill Estate, the trapping location of the positive African green monkey (Chlorocebus aethiops sabaeus) in 2019. All, but Wingfield, rivers leading to Old Town are now tapped near the source at $>300 \mathrm{~m}$ above sea level. However, the ghauts continue to have water during heavy rainfall periods.

other countries. No $S$. mansoni eggs have been detected in NHP feces (personal communication with the diagnostic laboratory on St. Kitts and unpublished data of the authors). In 2015, 94 fecal samples from recently trapped NHPs were submitted to the Natural History Museum in London (a WHO Collaborating Centre for the identification and characterization of schistosome strains and their snail intermediate hosts) for PCR testing. All samples were negative for the presence of S. mansoni eggs (unpublished data; feces supplied by and results communicated to J. Ketzis).

In 2019, 11 NHPs were trapped from the Green Hill Estate area (Figure 1), maintained in quarantine on St. Kitts for 4 months, and then transferred to quarantine in the United States, before dispersal to research facilities. As part of routine clinical examinations, feces from all NHPs were analyzed via fecal flotation with a focus on $T$. trichiura and protozoal infections while in quarantine on St. Kitts and treated accordingly. While in quarantine in the United States, also as a part of routine examinations, a direct smear was performed by a reference laboratory, and $S$. mansoni eggs were suspected in the feces of one NHP. No photographic documentation of the eggs was obtained, and no eggs could be found in subsequent subsamples of the fecal sample. The NHP was immediately treated, and feces at treatment time were collected and frozen for PCR and further analysis.

On arrival at the laboratory for PCR analysis, a decision was made to perform qualitative fecal sedimentation before DNA extraction with the feces. Three $S$. mansoni eggs were seen (representative one in Figure 2) in a subsample $(50 \mu \mathrm{L})$ of the sediment (approximately $1 \mathrm{~mL}$ ) and retrieved for DNA extraction and PCR analysis. DNA extraction was performed using the Qiagen DNeasy kit (Qiagen, Hilden, Germany). PCR was performed using a published protocol to amplify a 396-bp region of cox1 gene using the primers ASMIT1 and ASMIT2. ${ }^{17}$
Despite retrieval of eggs for DNA extraction, the PCR assay failed to amplify the intended region for S. mansoni. Reasons for this are unclear and require further investigation to determine if this method, used successfully for human diagnosis, can be effectively used in the NHP. Nevertheless, the unique morphology of eggs is confirmatory for S. mansoni diagnosis.

This finding of $S$. mansoni eggs in the feces of an NHP from St. Kitts is the first documentation of $S$. mansoni infection on St. Kitts since the 1950s. Although the NHP was no longer on St. Kitts at the time the infection was detected, given the quarantine facilities, use of potable water, and the management of the NHP, the only source of infection could have been while the NHP was resident on St. Kitts. The negative samples before shipment are likely due to the choice of a flotation method instead of the preferred sedimentation method for S. mansoni being used; however, even when sedimentation or direct smears are used, detection levels can be low. ${ }^{18,19}$

The origin of this infection is unknown. Potentially, S. mansoni has been recently reintroduced via the immigration or visitation of people from endemic Caribbean localities. Much of the Caribbean population is fluid with family members across islands traveling easily between them. Alternatively, S. mansoni transmission was either not eliminated or only below detectable limits and is still present in the human population. Another possibility is that S. mansoni has continued in a sylvatic cycle. This is in contradiction to what has been proposed regarding the NHP as final hosts. ${ }^{8}$ However, Green Hill Estate, the area from which this NHP was trapped, is in the vicinity of the head of the Cayon River, one of the previous sources of this parasite. Although the last testing and documentation of Biomphalaria spp. at the head of the Cayon River in 1958 were negative for S. mansoni and the only reported remaining population in the 1977 survey of Biomphalaria spp. was at the Fountain River, the 


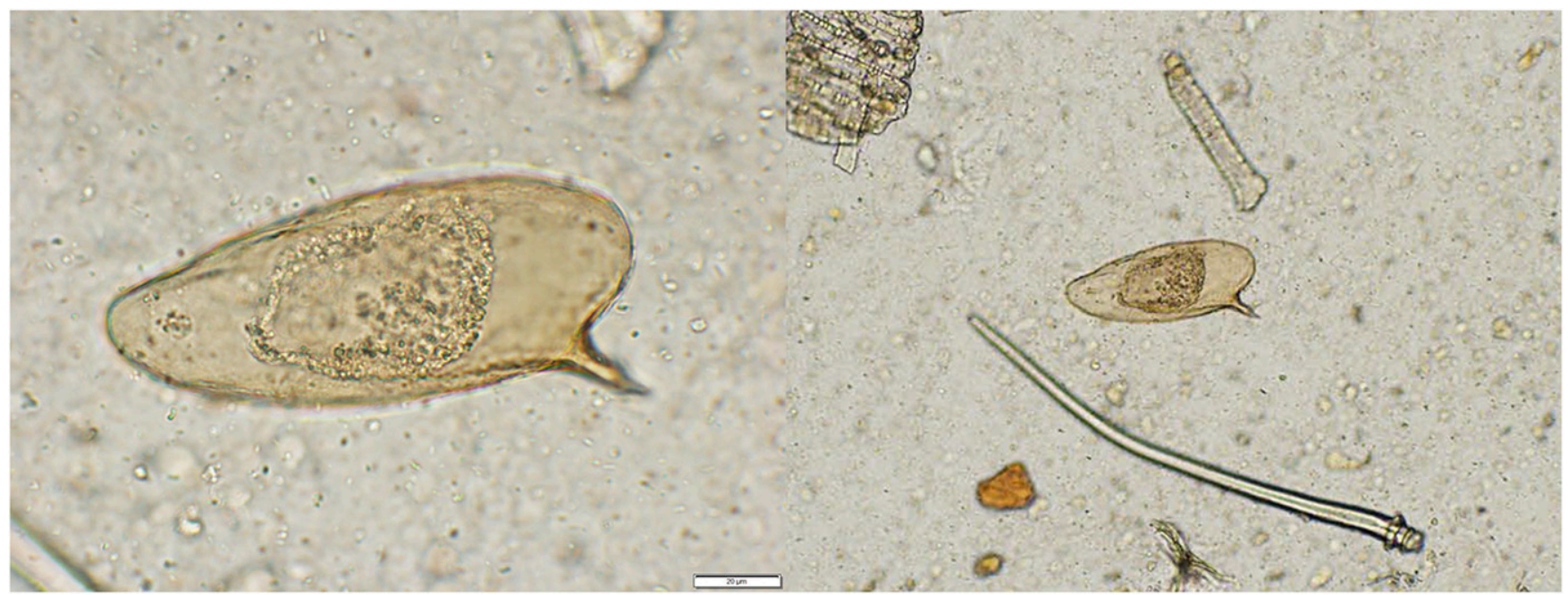

FIGURE 2. Schistosoma mansoni eggs isolated from the feces of the African green monkey (Chlorocebus aethiops sabaeus) in 2019.

Biomphalaria spp. population could have recovered at Cayon.

The finding of $S$. mansoni in the NHP raises important issues. First, the role of NHPs in perpetuating S. mansoni in a region might require reexamination, especially if elimination programs are to be effective. Second, this finding emphasizes the need for continued surveillance and monitoring of snail populations, even in areas where transmission has been stopped and elimination has been assumed.

Based on this report of S. mansoni, quarantined NHPs are being treated with praziquantel and parasite screening now includes sedimentation. In addition, snail surveys on St. Kitts are planned for the latter half of 2020 as well as serum (for antibody testing) and fecal collections (for egg identification) from NHPs trapped from Green Hill Estate and the Fountain River and Cayon River areas. Last, the St. Kitts and Nevis Medical Association and diagnostic laboratories on St. Kitts will be informed of this finding, although the risk of human infection and transmission is still likely low. In conclusion, the finding of this infection, in an NHP after over 50 years of transmission elimination in people in the locality, supports monitoring to ensure transmission does not reoccur if elimination of the intermediate host snail population has not been fully achieved.

Received April 12, 2020. Accepted for publication July 28, 2020.

Published online September 28, 2020.

Acknowledgment: We wish to thank Rutendo Mukaratirwa for production of the map included in Figure 1.

Disclosure: The St. Kitts nonhuman primate facility is accredited by the Canadian Council of Animal Care and the U.S. facility accredited by AAALAC and licensed for quarantine via the U.S. CDC. All fecal samples were collected as part of standard healthcare procedures.

Authors' addresses: Jennifer K. Ketzis, Ian Branford, and Arve Lee Willingham, One Health Center for Zoonoses and Tropical Veterinary Medicine, Ross University School of Veterinary Medicine, Basseterre, St. Kitts and Nevis, E-mails: jketzis@rossu.edu, ibranford@rossvet. edu.kn, and awillingham@rossvet.edu.kn. Manigandan Lejeune, Department of Population Medicine and Diagnostic Sciences, Animal Health Diagnostic Center, College of Veterinary Medicine, Cornell University, Ithaca, NY, E-mail: ml872@cornell.edu. Amy Beierschmitt,
Behavioural Science Foundation, Estridge Estate, St. Kitts and Nevis, E-mail: abeierschmitt@rossvet.edu.kn.

This is an open-access article distributed under the terms of the Creative Commons Attribution (CC-BY) License, which permits unrestricted use, distribution, and reproduction in any medium, provided the original author and source are credited.

\section{REFERENCES}

1. Lammie PJ, Lindo JF, Secor WE, Vasquez J, Ault SK, Eberhard $\mathrm{ML}, 2007$. Eliminating lymphatic filariasis, onchocerciasis, and schistosomiasis from the Americas: breaking a historical legacy of slavery. PLoS Negl Trop Dis 1: e71.

2. PAHO/WHO, 2014. Schistosomiasis Regional Meeting:Defining a Road Map toward Verification of Elimination of Schistosomiasis Transmission in Latin America and the Caribbean by 2020. Washington, DC: Pan American Health Organization. Available at: https://www.paho.org/hq/dmdocuments/2014/2014-chasch-regional-meeting-report.pdf. Accessed March 16, 2020.

3. WHO, 2013. WHO Schistosomiasis Progress Report 2001-2011 and Strategic Plan 2012-2020. Geneva, Switzerland: World Health Organization.

4. Rollinson D et al., 2013. Time to set the agenda for schistosomiasis elimination. Acta Trop 128: 423-440.

5. Ferguson FF, Richards CS, Sebastian ST, Buchanan IC, 1960. Natural abatement of Schistosoma mansoni in St. Kitts, British West Indies. Public Health 74: 261-265.

6. Hewitt R, Willingham AL, 2019. Status of schistosomiasis elimination in the Caribbean region. Trop Med Infect Dis 4: 24.

7. Prentice MA, 1980. Schistosomiasis and its intermediate hosts in the lesser Antillean Islands of the Caribbean. Bull Pan Am Health Organ 14: 258-268.

8. Bundy DAP, 1984. Caribbean schistosomiasis. Parasitology 89: 377-406.

9. Schneider MC et al., 2011. Elimination of neglected diseases in Latin America and the Caribbean: a mapping of selected diseases. PLoS Negl Trop Dis 5: e964.

10. Gaspard J, Usey MM, Fredericks-James M, Sanchez-Martin MJ, Atkins L, Campbell CH, Corstjens PLAM, van Dam GJ, Colley DG, Secor WE, 2020. Survey of schistosomiasis in Saint Lucia: evidence for interruption of transmission. Am J Trop Med Hyg 102: 827-831.

11. Cameron TWM, 1928. A new definitive host for Schistosoma mansoni. J Helminthol 6: 219-222.

12. Swellengrebel NH, Rijpstra AC, 1965. Lateral-spined schistosome ova in the intestine of a squirrel monkey from Suriname. Trop Geogr Med 17: 80-84. 
13. Alarcón de Noya B, Pointier JP, Colmenares $C$, Théron A, Balzan C, Cesari IM, González S, Noya O, 1997. Natural Schistosoma mansoni infection in wild rats from Guadeloupe: parasitological and immunological aspects. Acta Trop 68: 11-21.

14. Dore KM, 2017. Vervets in the Caribbean. Fuentes A, ed. The International Encyclopedia of Primatology. Hoboken, NJ: John Wiley \& Sons. Available at: https://doi.org/10.1002/ 9781119179313.wbprim0169.

15. Dore KM, 2013. An Anthropological Investigation of the Dynamic Human-Vervet Monkey Interface in St. Kitts, West Indies. PhD Thesis, University of Wisconsin-Milwaukee, Milwaukee, WI.

16. Ritchie LS, Knight WB, Oliver-González J, Frick LP, Morris JM, Croker WL, 1967. Schistosoma mansoni infections in Cercopithecus sabaeus monkeys. J Parasitol 53: 1217-1224.
17. Stothard JR, Webster BL, Weber T, Nyakaana S, Webster JP, Kazibwe F, Kabatereine NB, Rollinson D, 2009. Molecular epidemiology of Schistosoma mansoni in Uganda: DNA barcoding reveals substantial genetic diversity within Lake Albert and Lake Victoria populations. Parasitology 136: 1813-1824.

18. Meurs L, Brienen E, Mbow M, Ochola EA, Mboup S, Karanja DM, Secor WE, Polman K, van Lieshout L, 2015. Is PCR the next reference standard for the diagnosis of Schistosoma in stool? A comparison with microscopy in Senegal and Kenya. PLoS Negl Trop Dis 9: e0003959.

19. Guegan $\mathrm{H}$ et al., 2019. Real-time PCR for diagnosis of imported schistosomiasis. PLoS Negl Trop Dis 13: e0007711. 